Journal of Materials Chemistry B

Materials for biology and medicine www.rsc.org/MaterialsB

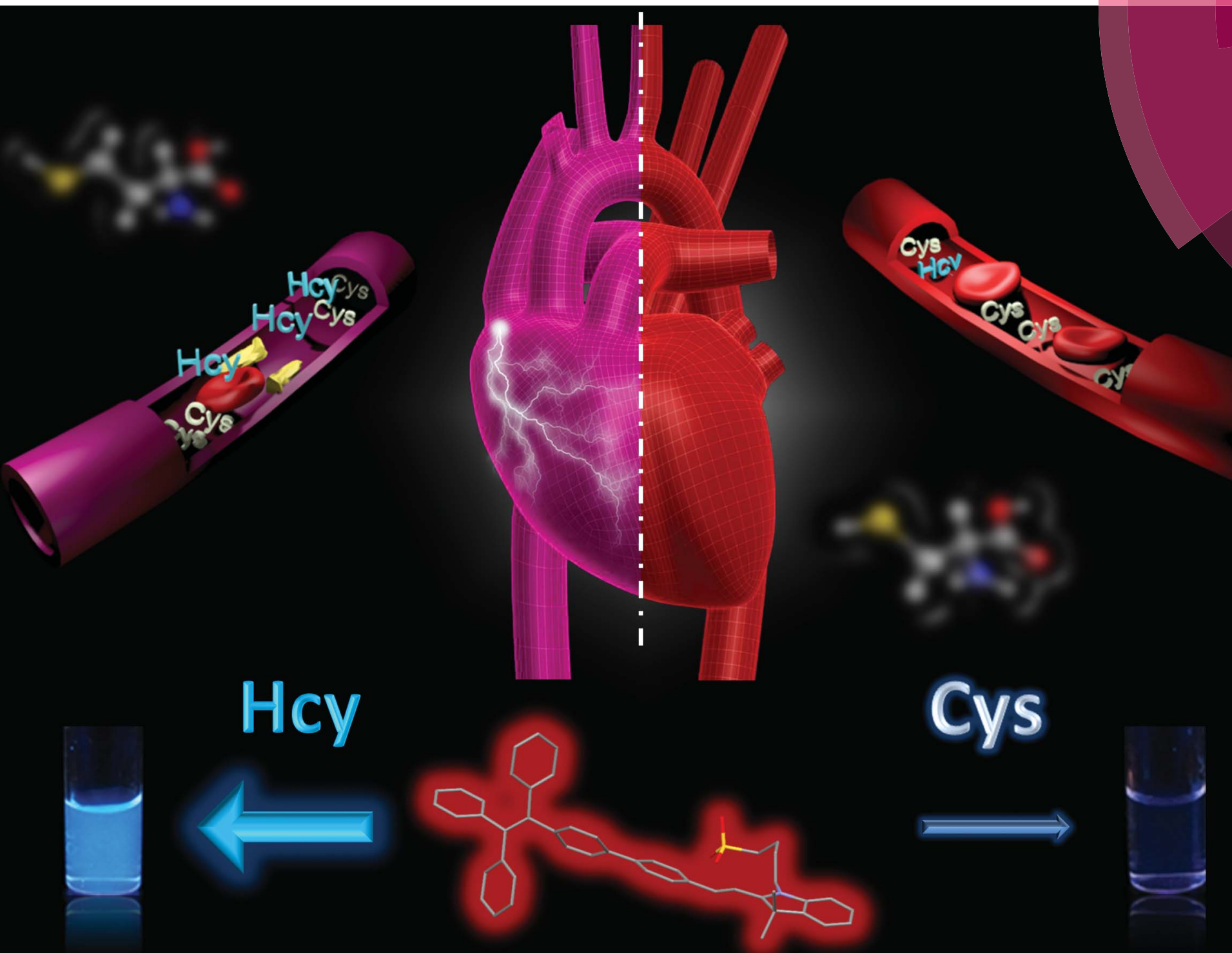


Received 8th April 2014

Accepted 29th April 2014

DOI: 10.1039/c4tb00551a

www.rsc.org/MaterialsB

\title{
Discrimination of homocysteine, cysteine and glutathione using an aggregation-induced- emission-active hemicyanine dye $\uparrow$
}

\author{
Sijie Chen, ${ }^{a}$ Yuning Hong, ${ }^{a}$ Jianzhao Liu, ${ }^{a}$ Nai-Wen Tseng, ${ }^{a}$ Yang Liu, ${ }^{a}$ Engui Zhao, ${ }^{a}$ \\ Jacky Wing Yip Lam ${ }^{a}$ and Ben Zhong Tang*abc
}

Elevated levels of homocysteine (Hcy) in blood are indicative of many high risk cardiovascular and neurodegenerative diseases. Thus, development of highly efficient and selective dyes for monitoring Hcy levels has attracted much attention. This paper reports the utilization of TPE-Cy, an aggregationinduced-emission active hemicyanine dye, as a probe for the detection of Hcy. More interestingly, this dye shows high selectivity to Hcy over cysteine, glutathione and other amino acids in weakly basic buffer solution.

\section{Introduction}

Thiol containing small biomolecules, including cysteine (Cys), homocysteine (Hcy) and glutathione (GSH), play a pivotal role in many biological systems. ${ }^{1}$ For example, Cys is the only amino acid with a thiol functional group that serves as a unique unit in protein construction, enzyme active sites and cofactors. ${ }^{1 a-c}$ On the other hand, GSH, a Cys containing tripeptide, is the most abundant free thiol in cells and is essential in maintaining the redox homeostasis in the intracellular environment. ${ }^{1{ }^{e f}}$ Recently, much research interest has been paid to Hcy because of its special role as a biomarker in many diseases. ${ }^{2}$ For instance, an elevated level of Hcy in the blood is a strong indicator for cardiovascular diseases, ${ }^{2 a, b}$ stroke and arteries or venous thrombosis. ${ }^{2 c}$ In addition, Hcy has also been reported to be associated with neurodegenerative disorders, ${ }^{2 d}$ such as neural tube defects, Alzheimer's disease ${ }^{2 g}$ and other cognitive impairments. ${ }^{2 h}$ Therefore, a tremendous amount of effort has been put into the development of efficient methods for monitoring Hcy levels for the early stage detection of these diseases.

For Hcy detection, one of the most common analytical techniques is liquid chromatography (LC). ${ }^{3}$ Although this method

${ }^{a}$ Division of Biomedical Engineering, Department of Chemistry, Institute for Advanced Study, Division of Life Science, State Key Laboratory of Molecular Neuroscience, and Institute of Molecular Functional Materials, The Hong Kong University of Science \& Technology, Clear Water Bay, Kowloon, Hong Kong, China. E-mail: tangbenz@ust. hk; Fax: +852-2358-1594; Tel: +852-2358-7375

${ }^{b}$ Guangdong Innovative Research Team, SCUT-HKUST Joint Research Laboratory, State Key Laboratory of Luminescent Materials and Device, South China University of Technology, Guangzhou, 510640, China

'HKUST-Shenzhen Research Institute, No. 9 Yuexing 1st RD, South Area, Hi-tech Park, Nanshan, Shenzhen, 518057, China

$\dagger$ Electronic supplementary information (ESI) available: ${ }^{13} \mathrm{C}$ NMR spectra. See DOI: $10.1039 / \mathrm{c} 4 \mathrm{tb} 00551 \mathrm{a}$ offers high accuracy, it typically requires staff with a certain skill level, intensive labour, and sophisticated instrument for the analysis, which makes it unsuitable for on-site trials and household testing. Fluorescent sensors, on the other hand, possess many advantages, such as superior sensitivity, simple manipulation and low cost, ${ }^{4}$ representing an alternative platform for Hcy detection. As a result, much effort has been devoted to the design and fabrication of fluorescent sensors for thiol recognition. ${ }^{5}$ Most of the sensing processes are designed based on the reactions that utilize strongly nucleophilic thiol groups, for example, through covalent bondings with olefins via Michael addition $^{5 f}$ or with carbonyls via heterocycle formation. ${ }^{5 b-d}$ Because all Hcy, Cys, and GSH contain thiol moieties, it is quite difficult to differentiate among these molecules using the reaction based fluorescent sensors. In particular, Hcy is a homologue of Cys with only one additional methylene $\left(-\mathrm{CH}_{2}-\right)$ group; the differentiation of these two is very challenging. Only a few cases have been reported for the selective detection of Hcy over Cys and GSH. ${ }^{6}$ Strongin and coworkers have developed a system for Hcy and Cys discrimination based on the differences in the cyclization rate of the two analytes with the probe. ${ }^{6 a}$ Huang and coworkers have designed an iridium(III) complex which shows relatively high specificity towards $\mathrm{Hcy}_{{ }^{6}}{ }^{6 b}$ Nevertheless, the sensing process of these probes is carried out in a media with large portion of organic solvents, which dramatically limits their application especially in biological systems.

Recently, an aggregation-induced-emission (AIE) active hemicyanine dye, TPE-Cy, has been developed by our group. ${ }^{7,8}$ This dye is sensitive toward $\mathrm{pH}$ changes: under basic conditions, the nucleophilic $\mathrm{OH}^{-}$ion can attach to the $\mathrm{C} 1$ site (Chart 1) and disrupt the conjugation of the tetraphenylethene (TPE) unit with the cyanine moiety, thus shifting the emission from red to blue. This proposed mechanism is supported by NMR and HRMS analyses. Thiol is a stronger nucleophile than the 


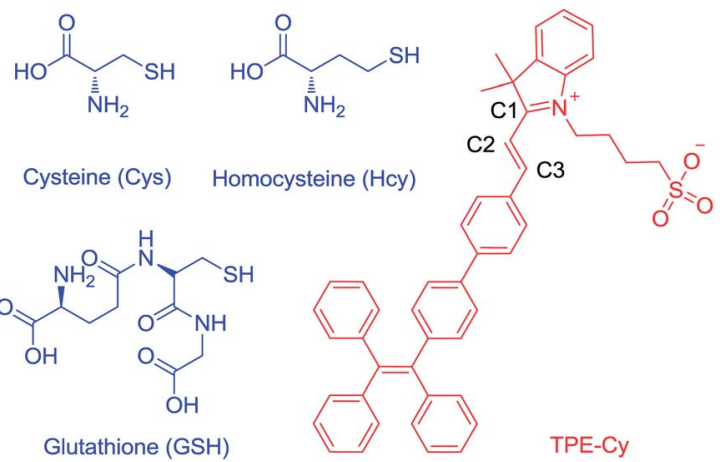

Chart 1 Structure of Cys, Hcy, GSH and TPE-Cy.

hydroxyl group and could also react with electrophilic TPE-Cy, changing its emission color. ${ }^{9}$ In this work, the feasibility of using TPE-Cy in biothiol detection is investigated, which demonstrates the specificity of the dye for Hcy recognition.

\section{Experimental section}

\section{Materials}

Buffer solution with $\mathrm{pH} 8$ was purchased from Merck. Homocysteine is purchased from TCI. Cysteine, glutathione, and other amino acids are purchased from Sigma-Aldrich.

\section{Instrumentation}

${ }^{1} \mathrm{H}$ and ${ }^{13} \mathrm{C}$ NMR spectra were measured on a Bruker AV 400 NMR spectrometer or a Bruker ARX 300 NMR spectrometer using DMSO- $d_{6}$, or $\mathrm{D}_{2} \mathrm{O}$ as the solvent and tetramethylsilane (TMS) as the internal reference. UV absorption spectra were recorded on a Milton Ray Spectronic 3000 array spectrophotometer. Photoluminescence (PL) spectra were recorded on a Perkin-Elmer LS 55 spectrofluorometer.

\section{Sample preparation}

TPE-Cy was synthesized according to previously reported procedures. $^{7}$ Different concentrations of Hcy, Cys, GSH or other amino acids were first dissolved in $\mathrm{pH} 8$ buffer and prepared by serial dilution. TPE-Cy ( $1 \mathrm{mM}$ in DMSO) stock solution was then added to the solution containing the analytes. The finial concentration of DMSO is $1 \%$. Steady state fluorescent measurement was taken 5 minutes after adding TPE-Cy.

\section{Characterization data}

Characterization data of Cys: ${ }^{1} \mathrm{H}$ NMR (400 MHz, $\left.\mathrm{D}_{2} \mathrm{O}\right), \delta$ (TMS, ppm): 3.98 (t, 1H), 3.12-2.99 (m, 2H). ${ }^{13} \mathrm{C}$ NMR (100 MHz, D $\left.{ }_{2} \mathrm{O}\right)$, $\delta$ (TMS, ppm): 171.71, 6.10, 24.1.

Characterization data of Hcy: ${ }^{1} \mathrm{H}$ NMR (400 MHz, DMSO- $d_{6}$ ), $\delta$ (TMS, ppm): $3.23(\mathrm{t}, 2 \mathrm{H}), 2.56(\mathrm{t}, 1 \mathrm{H}), 2.01-1.91(1 \mathrm{H}), 1.85-1.77$ $(1 \mathrm{H}) .{ }^{13} \mathrm{C}$ NMR (100 MHz, DMSO- $\left.d_{6}\right), \delta$ (TMS, ppm): 169.1, 52.9, 35.6, 20.6.

Characterization data of TPE-Cy + Cys: ${ }^{1} \mathrm{H}$ NMR $(400 \mathrm{MHz}$, DMSO- $d_{6}$ ), $\delta$ (TMS, ppm): 7.59-7.52 (m, 4H), 7.45 (d, 2H), 7.16$7.10(\mathrm{~m}, 11 \mathrm{H}), 7.09-6.96(\mathrm{~m}, 10 \mathrm{H}), 6.67(1 \mathrm{H}), 6.60(\mathrm{~d}, 1 \mathrm{H}) .5 .36$ (q, 1H), 4.79 (dd, 1H), 4.04-4.03 (m, 1H), $3.90(\mathrm{t}, 1 \mathrm{H}), 3.11$ (dd, 2H), 3.01-2.867 (m, 3H), 1.67 (s, 4H), 1.58 (d, 3H), 1.29 (d, 3H). ${ }^{13} \mathrm{C}$ NMR (100 MHz, DMSO- $d_{6}$ ), $\delta$ (TMS, ppm): 169.6, 169.4, 168.9 , 154.0, 153.7, 144.9, 143.2, 142.3, 141.9, 140.81, 140.2, $138.0,137.5,131.3,130.8,130.7,128.2$, 128.0, 127.9, 127.7, 126.7, 126.7, 126.5, 125.8, 121.4, 118.2, 105.5, 94.1, 93.8, 79.2, 54.7, 54.7, 52.1, 51.4, 51.2, 46.5, 46.2, 44.2, 41.2, 32.0, 28.5, 28.5, 27.9, 25.5, 23.0, 22.9 .

Characterization data of TPE-Cy + Hcy: ${ }^{1} \mathrm{H}$ NMR $(400 \mathrm{MHz}$, DMSO- $\left.d_{6}\right), \delta$ (TMS, ppm): 7.58-7.44 (m, 7H), 7.15-7.09 (m, 12H), $7.04-6.97(\mathrm{~m}, 10 \mathrm{H}), 6.66(\mathrm{t}, 1 \mathrm{H}), 6.59(\mathrm{~d}, 1 \mathrm{H}), 5.23(\mathrm{~d}, 1 \mathrm{H}), 4.74$ $(\mathrm{d}, 1 \mathrm{H}), 2.57(\mathrm{t}, 6 \mathrm{H}), 2.05-1.96(\mathrm{~m}, 5 \mathrm{H}), 1.93-1.86(\mathrm{~m}, 4 \mathrm{H}), 1.64$ (s, 4H), 1.57 (s, 4H), 1.31-1.25 (m, 4H), $1.16(\mathrm{~s}, 1 \mathrm{H}) .{ }^{13} \mathrm{C}$ NMR (100 MHz, DMSO- $d_{6}$ ), $\delta$ (TMS, ppm): 170.3, 170.1, 145.0, 143.2, 143.2 , 142.2, 140.8, 140.2, 137.7, 137.5, 131.3, 130.7, 128.1, 128.0, 127.8, 127.6, 126.7, 126.6, 126.5, 125.8, 118.1, 52.3, 51.3, $45.1,44.1,35.3,28.6,28.5,27.7,22.7,20.3$.

\section{Results and discussion}

\section{Responses of TPE-Cy to Hcy and Cys}

We first examine the reaction between TPE-Cy and Hcy. From the UV-vis spectra, TPE-Cy exhibits two absorption peaks at $\sim 440\left(A_{440}\right)$ and $\sim 330 \mathrm{~nm}\left(A_{330}\right)$ in $\mathrm{pH} 8$ buffer that can be attributed to the entire molecule and the TPE unit, respectively (Fig. 1). When Hcy is added to the solution, the absorption peak at $440 \mathrm{~nm}$ decreased dramatically while the counterpart at 330 $\mathrm{nm}$ increased, which is very similar to the phenomenon observed when a strong base is added. The change in TPE-Cy absorption indeed indicates the disruption of the conjugation between cyanine and TPE and the formation of new chemical species upon reaction with Hcy.

Similar to absorption spectra, the emission spectra of TPE-Cy changed upon the addition of Hcy (Fig. 2A). In pH 8 buffer, a weak red emission peak is predominant. Increasing the concentration of Hcy gradually weakens the intensity of this red emission peak at $615 \mathrm{~nm}$ and, on the other hand, promotes the emergence and enhancement of a new blue peak at $465 \mathrm{~nm}$. Since Cys also carries a thiol group, it can also react with TPE-Cy

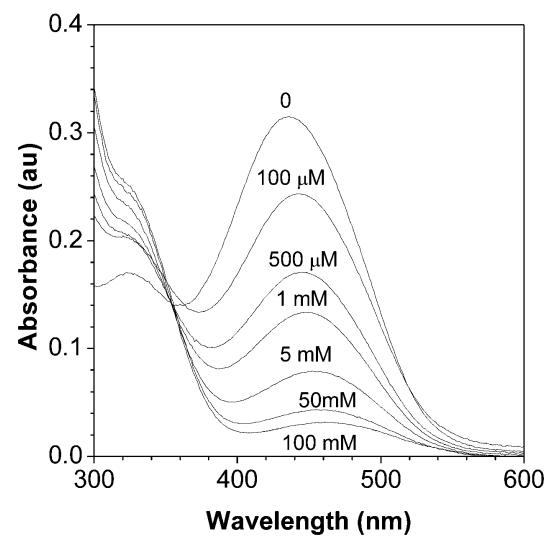

Fig. 1 Absorption spectra of $10 \mu \mathrm{M}$ TPE-Cy in the presence of different concentrations of Hcy in $\mathrm{pH} 8$ buffer. 

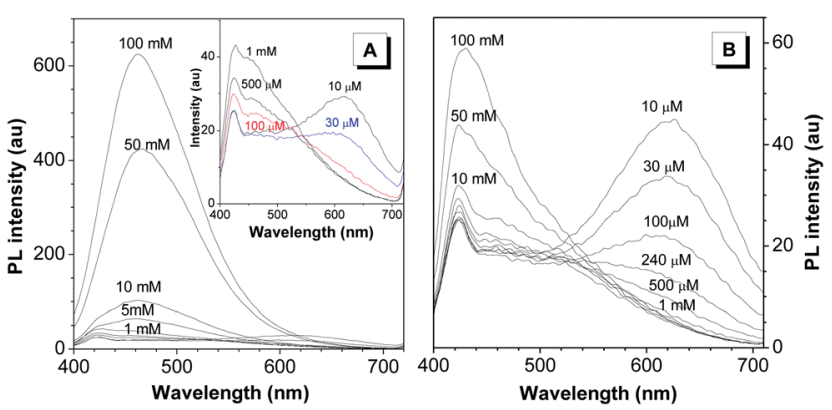

Fig. 2 PL spectra of TPE-Cy in $\mathrm{pH} 8$ buffer with different concentrations of (A) Hcy and (B) Cys. [TPE-Cy] $=10 \mu \mathrm{M}$.

and induce a similar change in its emission spectra when the Cys concentration is above $10 \mathrm{mM}$ (Fig. 2B).

The above observations suggest that both Hcy and Cys can react with TPE-Cy, however, the response to Hcy is much more pronounced than Cys. The high sensitivity and selectivity to Hcy can be clearly seen from the ratiometric changes in the absorption or emission of TPE-Cy (Fig. 3). The ratio of the absorption peak at 330 and $440 \mathrm{~nm}\left(A_{330} / A_{440}\right)$ is increased from 0.54 in pure buffer solution to 13.6 in the presence of $100 \mathrm{mM}$ Hcy but only 3.2 with the same concentration of Cys (Fig. 3A). When the analyte concentration is down to $100 \mu \mathrm{M}$, there is 1.6 times increment of the $A_{330} / A_{440}$ value for Hcy but almost indiscernible for Cys. The detection limit of TPE-Cy for Hcy is calculated to be $12 \mu \mathrm{M}$. Note that the physiological level of Hcy in blood is lower than $15 \mu \mathrm{M}$, above which it is considered as hyperhomocysteinemia. ${ }^{2 b}$ Under the same conditions, the detection limit for Cys is as high as $73 \mu \mathrm{M}$.
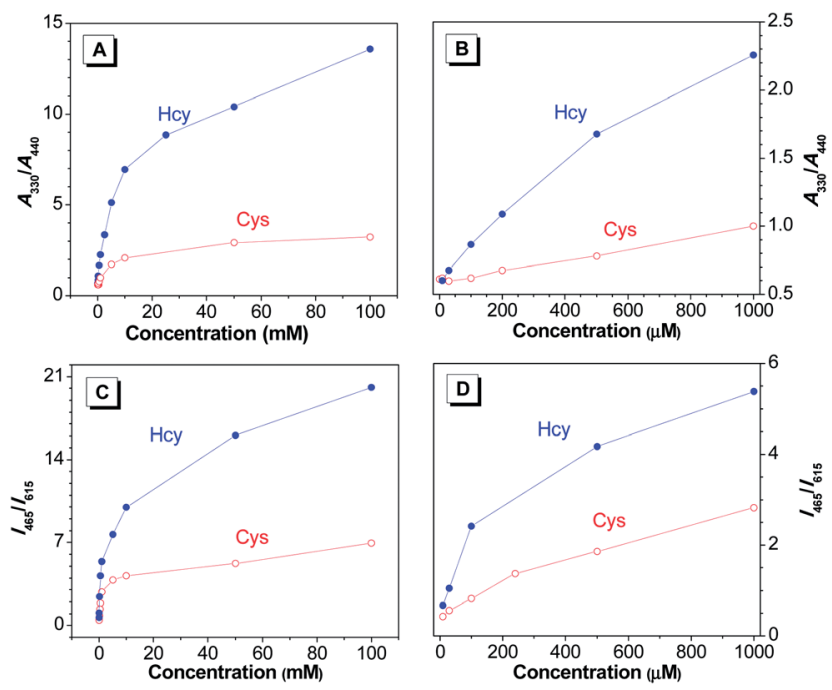

Fig. 3 (A) Plots of $A_{330} / A_{440}$ versus the concentration of Hcy or Cys, where $A_{330}$ and $A_{440}$ are the absorption values at wavelengths of 330 and $440 \mathrm{~nm}$, respectively. (B) The magnified range from 0 to $1 \mathrm{mM}$ of panel A. (C) Plots of $I_{465} / I_{615}$ versus the concentration of Hcy or Cys. $I_{465}$ and $I_{615}$ are the emission intensities at wavelengths of $465 \mathrm{~nm}$ and $615 \mathrm{~nm}$, respectively. (D) The magnified range from 0 to $1 \mathrm{mM}$ of panel C. [TPE-Cy] $=10 \mu \mathrm{M} ; \lambda_{\mathrm{ex}}=380 \mathrm{~nm}$. Tests are taken in $\mathrm{pH} 8$ buffer.
In terms of fluorescence, the ratio of the blue and red emission $\left(I_{465} / I_{615}\right)$ also demonstrates the sensitivity and selectivity of TPE-Cy towards Hcy (Fig. 3B). With $100 \mu \mathrm{M}$ Hcy, the $I_{465} / I_{615}$ value reaches 2.4 , which is 3 times higher than the value with the same concentration of Cys. This ratio is further increased to 20.4 when the Hcy concentration is $100 \mathrm{mM}$, while for Cys, the highest $I_{465} / I_{615}$ value is only 6.9. These results indicate that TPE-Cy shows higher response in both absorption and emission change toward Hcy over Cys.

\section{Selectivity test}

The selectivity of TPE-Cy to Hcy over other amino acids and GSH is then investigated. As shown in Fig. 4, the increment of the blue to red emission ratio induced by Hcy is much larger than that triggered by Cys and GSH, as well as other amino acids. The selectivity of TPE-Cy to Hcy can be clearly seen from the photos taken under UV irradiation. TPE-Cy shows red emission in buffer solution (blk) without any amino acid and the intensity of this red emission is slightly weakened when $100 \mathrm{mM} \mathrm{GSH}$ is added. On the other hand, when the same amount of Cys is introduced to the buffer solution, the emission color is changed from red to weak blue and intense blue emission is observed when Cys was replaced with Hcy. All these changes in emission color and intensity can be easily distinguished by naked eyes. With all other amino acids, the emission remains almost unchanged as the blank TPE-Cy solution.

\section{Mechanistic study}

The phenomenon of biothiol triggered emission changes of TPE-Cy prompts us to investigate the underlying working mechanism. The above absorption and emission behaviours all suggest a chemical reaction between TPE-Cy and Hcy and the reaction product is thus fully analysed by NMR spectroscopy. Retrostructural analysis of TPE-Cy suggests that the potential reactive site could be the carbon atoms labeled with $\mathrm{C} 1, \mathrm{C} 2$, and C3 in the chemical structure shown in Scheme 1. The thiol group can act as a nucleophile to attack the electron-deficient $\mathrm{C} 1, \mathrm{C} 2$, or $\mathrm{C} 3$, resulting in the breakage of the conjugation and switch the emission of TPE-Cy from red to blue. Considering the $\alpha, \beta$-unsaturated iminium moiety in TPE-Cy, C1 and C3 are the

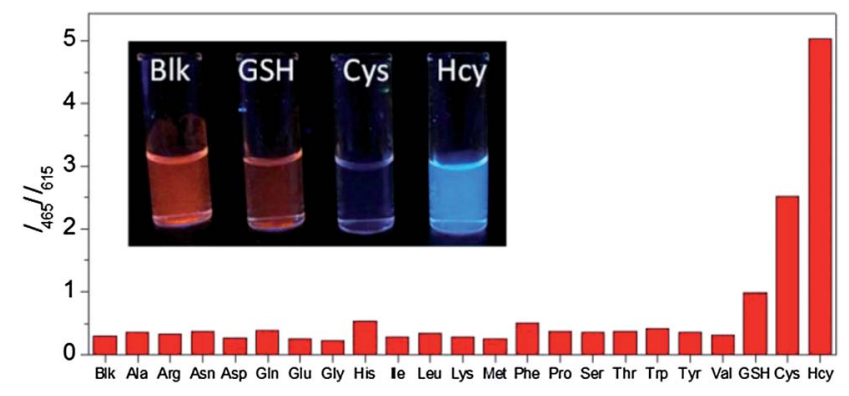

Fig. 4 Emission ratio $/ 465 / /_{615}$ of TPE-Cy in the absence or presence of $1 \mathrm{mM}$ different amino acids or GSH in pH buffer. [TPE-Cy] $=10 \mu \mathrm{M}$; $\lambda_{\text {ex }}=380 \mathrm{~nm}$. Inset: photo of $10 \mu \mathrm{M}$ TPE-Cy in blank buffer or $100 \mathrm{mM}$ GSH, Cys and Hcy (from left to right) under a handheld UV lamp illumination. 


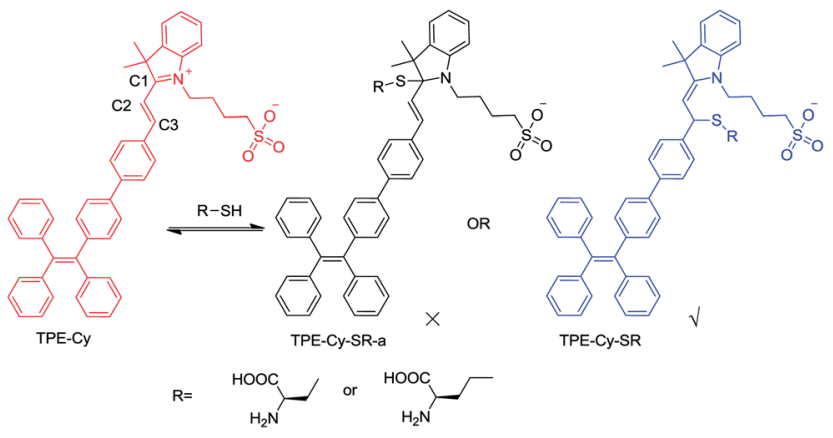

Scheme 1 Proposed mechanism of the reaction between TPE-Cy and biothiols.

possible sites for the addition reaction to occur. The 1,2-addition mode at C1 gives TPE-Cy-SR-a while the 1,4-addtion mode at C3 generates TPE-Cy-SR (Scheme 1). In our previous work using TPE-Cy as a $\mathrm{pH}$ sensor, we have proposed that the nucleophilic $\mathrm{OH}^{-}$prefers to attack the $\mathrm{C} 1$ position and result in pH-switched red/blue emission. ${ }^{7}$ Based on the hard soft acid base theory, ${ }^{10}$ we believe that the hard nucleophile like $\mathrm{OH}^{-}$ prefers to react at the $\mathrm{C} 1$ position while the soft nucleophile like RSH would prefer to react at the C3 site.

To verify our hypothesis, NMR analyses are used. Upon addition of Cys or Hcy, most of the proton signals in the aromatic region are shifted upfield, presumably due to the absence of the electron-withdrawing iminium functional group, except for protons on phenyl groups that form the TPE core which remains unshifted (Fig. 5). Closer examination of the resulting spectra reveals the absence of the olefin protons for

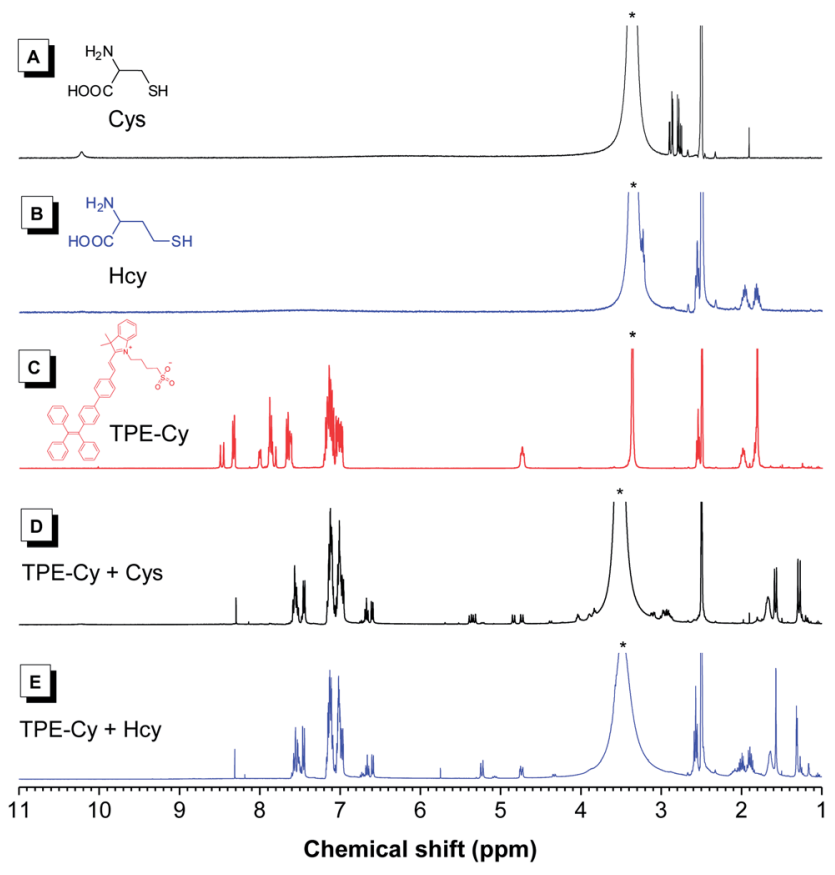

Fig. $5{ }^{1} \mathrm{H}$ NMR spectra of (A) Cys, (B) Hcy, (C) TPE-Cy, (D) TPE-Cy with Cys and (E) TPE-Cy with Hcy in DMSO- $d_{6}$. The water peaks are marked with asterisks. the $\alpha, \beta$-unsaturated iminium molecule ( 7.83 and 8.48 ) and a new set of signals corresponding to the enamine olefin (5.0-5.5) and benzylic allylic proton (4.5-5.0) appeared, indicating that the reaction of Cys or Hcy with TPE-Cy proceeds in a 1,4-addition fashion. It is clear that the excessive Hcy/Cys can attack the C3 position of the TPE-Cy molecule, which breaks the conjugation, and hence switches the red emission into blue. Compared with Cys, Hcy has one additional methylene $\left(-\mathrm{CH}_{2}-\right)$ group, the longer spacer provides less steric effect, which could facilitate the addition reaction. On the other hand, presumably due to the greater steric hindrance, the tripeptide biothiol GSH has the lowest reactivity towards TPE-Cy compared to Cys or Hcy. The ${ }^{13} \mathrm{C}$ NMR spectra of these reactions are provided in the ESI (Fig. S1†).

In addition to the reactivity differences, we believe that the AIE property of TPE-Cy also plays an important role in its different responses to Hcy and Cys. Upon addition reaction with biothiol, the positively charged nitrogen on TPE-Cy is neutralized which will increase the hydrophobicity of the resulting product. Because Hcy is more hydrophobic than Cys, the product from the TPE-Cy and Hcy reaction would have higher tendency to aggregate in the aqueous buffer solution. Because of its AIE property, the aggregate formation restricts the intramolecular rotations, upon which, the radiative decay will be populated and the fluorescence will be enhanced. The collective effect makes the TPE-Cy-Hcy adduct more emissive than TPE-Cy-Cys.

In summary, a red-emission hemicyanine AIE-active dye, TPE-Cy, has been utilized for the discrimination of Hcy, Cys and GSH. TPE-Cy possesses high selectivity toward Hcy over other amino acids, including the thiol containing Cys and the tripeptide, GSH. Both the absorption and fluorescence emission of TPE-Cy are sensitive to Hcy. In the presence of Hcy, the red emission of TPE-Cy in weakly alkalized buffer is suppressed while a blue emission appears. Cys, although can also decrease the red emission of TPE-Cy, cannot promote the blue emission as much as Hcy and results in a weak blue emission. NMR analysis suggests that the thiol group can attack the double bond between the TPE unit and the cyanine unit of TPE-Cy through a 1,4-addition fashion which is facilitated by the less sterically hindered Hcy. Because of the steric hindrance, GSH is the least reactive and no obvious difference in terms of fluorescence can be observed of TPE-Cy with GSH. The collective effect of the reaction and the AIE property of the dye enables its sensitivity and selectivity to Hcy over other biothiols.

\section{Acknowledgements}

This work was partially supported by the National Basic Research Program of China (973 Program; 2013CB834701), the Research Grants Council of Hong Kong (604711, 604913, HKUST2/CRF/10 and N_HKUST620/11), the Innovation and Technology Commission (ITCPD/17-9) and the University Grants Committee of Hong Kong (AoE/P-03/08). B.Z.T. thanks the support from the Guangdong Innovative Research Team Program (201101C0105067115). 


\section{Notes and references}

1 (a) B. Albers, D. Bray, K. Hopkin, A. Johnson, J. Lewis, M. Raff, K. Roberts and P. Walter, Essential Cell Biology, Garland Science, New York, 3rd edn, 2009; (b) S. M. Marino and V. N. Gladyshev, J. Mol. Biol., 2010, 404, 902; (c) S. A. Lipton, Y.-B. Choi, H. Takahashi, D. Zhang, W. Li, A. Godzik and L. A. Bankston, Trends Neurosci., 2002, 25, 474; (d) Z. Cheng, X. Yang and H. Wang, Curr. Hypertens. Rev., 2009, 5, 158; (e) V. I. Lushchak, J. Amino Acids, 2012, 2012, 736837; (f) G. Wu, Y. Z. Fang, S. Yang, J. R. Lupton and N. D. Turner, J. Nutr., 2004, 134, 489.

2 (a) D. S. Wald, M. Law and J. K. Morris, Br. Med.J., 2002, 325, 1202; (b) A. Majors, L. A. Ehrhart and E. H. Pezacka, Arterioscler., Thromb., Vasc. Biol., 1997, 17, 2074; (c) R. C. Austin, S. R. Lentz and G. H. Werstuck, Cell Death Differ., 2004, 11, S56; (d) R. Diaz-Arrastia, Arch. Neurol., 2000, 57, 1422; (e) Q. Xiao, F. Shang, X. Xu, Q. Li, C. Lu and J.-M. Lin, Biosens. Bioelectron., 2011, 30, 211; $(f)$ R. Carmel and D. W. Jacobsen, Homocysteine in Health and Disease, Cambridge University Press, Cambridge, 2001; $(g)$ S. Seshadri, A. Beiser, J. Selhub, P. F. Jacques, I. H. Rosenberg, R. B. D'Agostino, P. W. F. Wilson and P. A. Wolf, N. Engl. J. Med., 2002, 346, 476; (h) A. L. Miller, Alternative Med. Rev., 2003, 8, 7.

3 (a) H. Refsum, A. D. Smith, P. M. Ueland, E. Nexo, R. Clarke, J. McPartlin, C. Johnston, F. Engbaek, J. Schneede, C. McPartlin and J. M. Scott, Clin. Chem., 2004, 50, 3; (b) M. I. Amores-Sánchez and M. A. Medina, Clin. Chem. Lab. Med., 2000, 38, 199; (c) W. Sawuła, Z. Banecka-Majkutewicz, L. Kadziński, J. Jakóbkiewicz-Banecka, G. Wegrzyn, W. Nyka and B. Banecki, Acta Biochim. Pol., 2008, 55, 119; (d) K. Kuśmierek, R. Głowacki and E. Bald, Anal. Bioanal. Chem., 2006, 385, 855.

4 (a) Chemosensors: Principles, Strategies, and Applications, ed. B. Wang and E. V. Anslyn, John Wiley \& Sons Inc., New Jersey, 2011; (b) I. Jonhson and M. T. Z. Spence, The
Molecular Probes Handbook, Invitrogen Corp, Carlsbad, 11th edn, 2010; (c) X. Zhang, Z. Yang, Z. Chi, M. Chen, B. Xu, C. Wang, S. Liu, Y. Zhang and J. Xu, J. Mater. Chem., 2010, 20, 292; (d) X. Zhang, Z. Chi, B. Xu, H. Li, W. Zhou, X. Li, Y. Zhang, S. Liu and J. Xu, J. Fluoresc., 2011, 21, 133; (e) X. Li, X. Zhang, Z. Chi, X. Chao, X. Zhou, Y. Zhang, S. Liu and J. Xu, Anal. Methods, 2012, 4, 3338; (f) X. Zhang, M. Liu, B. Yang and Y. Wei, Colloids Surf., B, 2013, 112, 81; (g) Y. Hong, L. Meng, S. Chen, C. W. T. Leung, L.-T. Da, M. Faisal, D.-A. Silva, J. Liu, J. W. Y. Lam, X. Huang and B. Z. Tang, J. Am. Chem. Soc., 2011, 134, 1680.

5 (a) H. Y. Shiu, M. K. Wong and C. M. Che, Chem. Commun., 2011, 47, 4367; (b) L. Xiong, Q. Zhao, H. Chen, Y. Wu, Z. Dong, Z. Zhou and F. Li, Inorg. Chem., 2010, 49, 6402; (c) X. Zhang, X. Ren, Q.-H. Xu, K. P. Loh and Z.-K. Chen, Org. Lett., 2009, 11, 1257; (d) W. Lin, L. Long, L. Yuan, Z. Cao, B. Chen and W. Tan, Org. Lett., 2008, 10, 5577; (e) J. Shao, H. Sun, H. Guo, S. Ji, J. Zhao, W. Wu, X. Yuan, C. Zhang and T. D. James, Chem. Sci., 2012, 3, 1049; (f) S. Sreejith, K. P. Divya and A. Ajayaghosh, Angew. Chem., Int. Ed., 2008, 47, 7883.

6 (a) X. Yang, Y. Guo and R. M. Strongin, Angew. Chem., Int. Ed., 2011, 50, 10690; (b) H. Chen, Q. Zhao, Y. Wu, F. Li, H. Yang, T. Yi and C. Huang, Inorg. Chem., 2007, 46, 11075.

7 S. Chen, J. Liu, Y. Liu, H. Su, Y. Hong, C. K. W. Jim, R. T. K. Kwok, N. Zhao, W. Qin, J. W. Y. Lam, K. S. Wong and B. Z. Tang, Chem. Sci., 2012, 3, 1804.

8 S. Chen, Y. Hong, Y. Liu, J. Liu, C. W. T. Leung, M. Li, R. T. K. Kwok, E. Zhao, J. W. Y. Lam, Y. Yu and B. Z. Tang, J. Am. Chem. Soc., 2013, 135, 4926.

9 G. T. Dempsey, M. Bates, W. E. Kowtoniuk, D. R. Liu, R. Y. Tsien and X. Zhuang, J. Am. Chem. Soc., 2009, 131, 18192.

10 (a) R. G. Pearson and J. Songstad, J. Am. Chem. Soc., 1967, 89, 1827; (b) J. March, Advanced Organic Chemistry, Reactions, Mechanisms and Structure, John Willey, Chichester, 4th edn, 1992. 